Herz 2014 · 39:421-422

DOI 10.1007/s00059-014-4101-6

Online publiziert: 25. April 2014

(c) Urban \& Vogel 2014

R.R. Tilz • K.-H. Kuck

Abteilung für Kardiologie, Asklepios Klinik St. Georg, Hamburg

\title{
Synkope und Schwindel
}

Synkopen und Schwindel sind sehr häufige Diagnosen und betreffen Patienten in jedem Lebensalter. So erleiden rund $40 \%$ aller Menschen mindestens einmal im Leben eine Synkope [1, 2, 3, 4]. Die Synkope ist eine der häufigsten Vorstellungsgründe in einer Notaufnahme. Rund 1-2\% aller Patienten in einer Notaufnahme werden aufgrund einer Synkope vorstellig. Die Ursachen der Synkope sind vielfältig und erfordern in vielen Fällen eine interdisziplinäre Abklärung. So wurden die aktuellen Leitlinien der Europäischen Gesellschaft für Kardiologie für die Diagnose und Therapie der Synkope in Zusammenarbeit mit vielen Fachgesellschaften erarbeitet [1]. Unter anderem haben internationale Fachgesellschaften der Geriater, Notfallmediziner, Internisten und Neurologen mitgewirkt. In diesem Themenheft haben wir uns bemüht, dieses komplexe Thema in Anlehnung an die aktuellen Leitlinien der Europäischen Gesellschaft für Kardiologie übersichtlich darzustellen.

Der erste Artikel aus unserer Arbeitsgruppe gibt einen Überblick über Epidemiologie, Definition, Klassifikation, $\mathrm{Pa}$ thophysiologie und Prognose der Synkope. In den aktuellen Leitlinien wurde die Definition der Synkope verbessert und die Klassifikation deutlich vereinfacht. Das erklärte Ziel war, über sämtliche Fachdisziplinen hinweg ein standardisiertes, einheitliches Klassifikationssystem und Vorgehen einzuführen, um die Patienten möglichst schnell der entsprechenden Fachrichtung und somit einer zielgerichteten Diagnostik und Therapie zuzuführen.

Im Artikel der hannoverschen Arbeitsgruppe beschreiben König et al. die klinische Abklärung und Risikostratifizierung der Synkope. Synkopen sind nicht nur bedeutsam, weil sie zu einer erheblichen Einschränkung der Lebensqualität führen, sondern weil sie in Abhängigkeit von Komorbidität und Ursache der Synkope mit einer erhöhten Mortalität assoziiert sein können. Von herausragender Bedeutung ist es, $\mathrm{Pa}$ tienten mit erhöhtem Mortalitätsrisiko zu identifizieren und unter stationären Bedingungen weiter abzuklären. Im Gegensatz dazu können Patienten mit einem geringen Risiko ambulant abgeklärt werden. Generell sind in den letzten Jahren zunehmend spezialisierte Einrichtungen, sog. „Synkopen-Units“ (Synkopeneinheiten) gegründet worden. Ziel dieser Einrichtungen ist eine spezialisierte, interdisziplinäre und gezielte Abklärung von Synkopen [5]. Zudem hat in den letzten Jahren der implantierbare Ereignisrekorder enorm an Bedeutung gewonnen. Er ermöglicht erstmals eine jahrelange Überwachung des Herzrhythmus und ist daher vor allem zum Nachweis oder Ausschluss einer rhythmogenen Ursache einer Synkope äußerst hilfreich.

Die Behandlung der Synkope wird von Andresen im dritten Artikel erläutert. Die Therapie ist abhängig von der vermuteten oder bekannten Ursache der Synkope. Eine kurative Therapie gibt es in vielen Fällen nicht. Die Behandlungsoptionen reichen von der Vermeidung reversibler Ursachen, dem Tragen elastischer Strümpfe, der Katheterablation bis zur Implantation von Schrittmachern oder Defibrillatoren. Eine medikamentöse Therapie zur Synkopenprävention ist aktuell nur in Sonderfällen indiziert. Zwar wurden viele Medikamente zur Synkopenprävention getestet, jedoch waren die Effekte der meisten Medikamente in den Studien enttäuschend. 
Bickel und Röther bereichern das Themenheft mit einem Artikel über Synkopen aus Sicht des Neurologen. Sie geben einen Überblick über wichtige neurologische Ursachen für Synkopen wie neurodegenerative Erkrankungen (z. B. Morbus Parkinson oder Polyneuropathien), welche über eine Störung autonomer Reflexe zu Synkopen führen können. Zudem beschreiben sie neurologische Differenzialdiagnosen der Synkopen wie Epilepsien, „drop attacks“ oder Durchblutungsstörungen im Basilarisstromgebiet.

Abgerundet wird das Themenheft durch drei spannende und lehrreiche Fälle von Athanasiadis und Sechtem aus Stuttgart.

Im Unterschied zum akuten Thoraxschmerz, welcher in deutschen Kliniken zunehmend in spezialisierten „Chest Pain Units“ abgeklärt wird, erfolgt die Synkopenabklärung derzeit meist noch nicht in spezialisierten Synkopeneinheiten. Aufgrund der mannigfaltigen Ursachen der Synkope, der Häufigkeit der Ereignisse sowie der damit verbundenen Kosten werden spezialisierte Einheiten mit Expertisen aus verschiedenen Fachrichtungen von den aktuellen Leitlinien empfohlen und erfreuen sich zunehmender Beliebtheit.

\section{Roland Richard Tilz \\ Karl-Heinz Kuck}

\section{Korrespondenzadresse}

\section{Dr. R.R. Tilz}

Abteilung für Kardiologie,

Asklepios Klinik St. Georg

Lohmühlenstraße 5,

20099 Hamburg

tilz6@hotmail.com

Interessenkonflikt. R. R. Tilz und K.-H. Kuck geben an, dass kein Interessenkonflikt besteht.

\section{Literatur}

1. Moya A, Sutton R, Ammirati F et al (2009) Guidelines for the diagnosis and management of syncope (version 2009). Eur Heart J 30:2631-2671

2. Soteriades ES, Evans JC, Larson MG et al (2002) Incidence and prognosis of syncope. N Engl J Med 347:878-885

3. Ganzeboom KS, Colman N, Reitsma JB et al (2003) Prevalence and triggers of syncope in medical students. Am J Cardiol 91:1006-1008, A8

4. Brignole M, Menozzi C, Del Rosso A et al (2000) New classification of haemodynamics of vasovagal syncope: beyond the VASIS classification. Analysis of the pre-syncopal phase of the tilt test without and with nitroglycerin challenge. Vasovagal Syncope International Study. Europace 2:66-76

5. Dagres N, Bongiorni MG, Dobreanu D et al (2013) Current investigation and management of patients with syncope: results of the European Heart Rhythm Association survey. Europace 15:18121815

\section{Neuer Schnelltest auf Nieren- versagen nach Herz-OP}

Durch einen Urintest kann eine beginnende Nierenschädigung bei Patienten nach herzchirurgischen Eingriffen früher erkannt werden als mit herkömmlichen Verfahren. Das zeigten Forscher der Universität Münster in einer Studie.

Sie untersuchten Patienten, die sich einem Eingriff unter Verwendung einer Herz-Lungen-Maschine unterziehen mussten. Die Wissenschaftler verglichen herkömmliche Nierenfunktionstests mit einem neuen, in den USA entwickelten Urintest, der die Proteine TIMP-2 und IGFBP-7 im Urin der Patienten nachweisen kann. Die Freisetzung dieser Proteine durch die Nierenzellen fungiert als eine Art Alarmsystem, das signalisiert, wenn tubuläre Epithelzellen unter akutem Stress stehen und die Gefahr eines akuten Nierenversagens gegeben ist.

Das Ergebnis der jetzt veröffentlichten Studie zeigt, dass eine Schädigung der Niere bereits vier Stunden nach einer Herz-Op. zuverlässig nachgewiesen werden kann. In weiteren Studien soll untersucht werden, ob die Verwendung des Urintests in Verbindung mit nierenschützenden Maßnahmen zu weniger Komplikationen und damit zu einem schnelleren Heilungsverlauf führen kann.

\section{Literatur}

Meersch M et. al (2013) Urinary TIMP-2 and IGFBP7 as early biomarkers of acute kidney injury and renal recovery following cardiac surgery. PLOS ONE. doi: 10.1371/journal.pone.0093460

Quelle:

Medizinische Fakultät der Universität Münster, www.campus.uni-muenster.de 